\title{
The art of conversation between plants and bacteria
}

\author{
Siva Linga Sasanka Velivelli
}

School of Biological, Earth \& Environmental Sciences, UCC

\section{Introduction}

Plant pests and pathogens have been a serious problem for farmers for many years and have been a major threat to plant health and food production. Farmers use synthetic chemicals to improve crop yields and to control plant pathogens from destroying their crops. Chemical-based fertilizers provide immediate relief, but their excessive use also causes severe environmental problems and can have adverse effects on groundwater, plants, animals and even entire ecosystems. Many countries have banned highly toxic chemicals which are harmful to the environment. One of the key challenges facing plant biologists is the development of new technologies and environmentally friendly alternatives to chemicals for combating crop diseases. An increase in world population has resulted in a reduction in agricultural land area. However, this area will be required to produce $50 \%$ more food by 2050 to feed the 10 billion people living on the planet.

Biological control plays an important role in agricultural systems. It is considered one of the most promising methods for safe crop-management practices and is one area of rapidly expanding research. Biological control is defined as the use of beneficial microorganisms, such as bacteria, to control plant diseases. Bacteria are microscopic, single-celled organisms present in all soil samples. Plant roots interact with the surrounding soil and support a range of microorganisms that can have either a harmful or beneficial effect on the plant. Nowadays, emphasis is placed on beneficial microorganisms which play a key role in soil structure maintenance, soil borne disease control and plant growth promotion activities. These beneficial microorganisms are known as plant growth-promoting rhizobacteria (PGPRs) ('Rhizo' means root).

Plant growth-promoting rhizobacteria (PGPRs) are free-living soil bacteria (bacteria capable of living in a wide range of natural environments) that colonize root surfaces and have the capacity to enhance plant growth directly or indirectly. PGPRs have gained worldwide importance over the last few years. These can reduce plant diseases without a negative impact on the environment unlike that caused by chemicals. Plants are exposed to attack from a wide variety of enemy organisms (insect pests, pathogenic bacteria and fungi) and stressful environmental conditions. In order to protect themselves, plants have defence mechanisms to fight against their invaders. As part of their defence mechanism, plants 
emit a range of volatile organic compounds (VOCs) from leaves, flowers and fruits into the atmosphere and from the roots into the soil. Volatile organic compounds (VOCs) are chemical compounds that generally have a high vapour pressure. These volatiles help in communication to the outside world and can carry both private and public messages and transmit information within a plant and potentially between plants. VOCs that are released from infected plants can serve as airborne signals and can be used as alarmcues. These airborne signals warn neighbouring plants about pathogen attack and elicit the activation of the defence mechanism thus allowing for a stronger response in future attacks.

How is the plant defence mechanism activated? It is through the use of plant growth promoting rhizobacteria. Rhizobacteria also release a wide variety of volatile organic compounds which stimulate plant growth and suppress the growth of plant pathogens via VOC emissions. Different rhizobacteria release different volatiles and these can serve as a mode of intra-and inter-species communication between plants and rhizobacteria. Some rhizobacteria release 2,3-Butanediol. This is a VOC which can promote plant growth and can interact with plants in many different ways by triggering a plant-mediated induced systemic resistance (ISR). ISR is defined as a state of enhanced defensive capacity developed by a plant against a broad spectrum of plant pathogens and pests. In recent years, plant scientists have become interested in the use of rhizosphere microorganisms that release a complex blend of volatile organic compounds in a way that can influence plant health and play a key role in the suppression of plant pathogens. In this present study, a commercial rhizobacterial strain, Bacillus subtilis FZB24, was used as a model to develop the system at the laboratory level for the early detection and selection of novel PGPRs that may have potential as biocontrol agents in crop production.

\section{My Research}

My research project (VALORAM -Valorizing (meaning- adding value or to assign value) Andean microbial diversity through sustainable intensification of potato-based farming systems (using rhizosphere isolated from the Andean potato)) aims at exploring the large biodiversity of existing soil microorganisms for their ability to antagonise (ability to suppress plant pathogens), promote growth and protect against disease in Andean traditional agriculture (In the Andes, potato plays a central role in livelihood systems and many people still use foot ploughs for farming). This could help to reduce excessive use of agrochemicals and support sustainable agriculture and environmental protection. This project was focussed on potato because of its global importance for small-scale farmers in the central Andean highlands (Ecuador, Peru and Bolivia).

A commercial rhizobacterial strain, B. subtilis FZB24 (using rhizosphere isolated from the Andean potato) was used as a model at the laboratory level for the early detection and 
selection of novel PGPRs through characterisation of an array of their volatile organic compounds.

A rhizobacterial strain was grown in a liquid culture to gather a rhizobacterial volatile, a solid phase microextraction fibre (SPME - a technique for the extraction of volatile organic chemical compounds from liquid), coated with polydimethylsiloxane was used. In this process, chemical vapours rise above the liquid test sample and stick to the fibre. The fibre (SPME) was then placed in the Gas chromatography machine coupled with mass spectrometry (GC/MS - It is a technique that can be used to separate and identify chemical compounds) to detect VOCs produced by the commercial rhizobacteria (Fig.1). i. vials inoculated with rhizobacteria, ii. Extraction of volatiles by SPME, iii. GC-MS analysis.
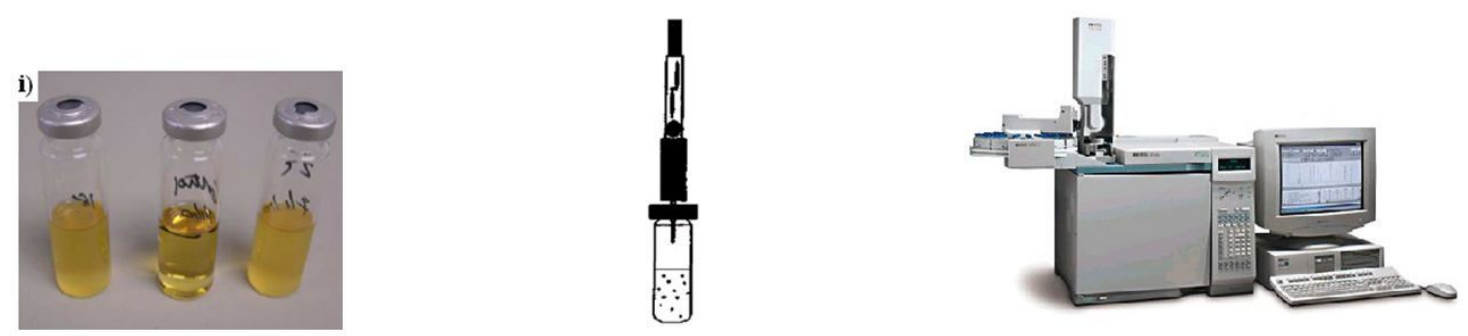

Figure 1: Analysis of rhizobacterial volatiles by SPME-GC/MS (Copyright: Shimadzu $\mathrm{GC} / \mathrm{MS}$ )

\section{Findings}

This research examines the detection of volatile organic compounds (VOCs) emitted from the commercial rhizobacterial strain, namely, B. subtilis FZB24. The GC-MS analysis identified different volatiles in laboratory conditions. Among these, 2,3 butanediol seem to be the most promising compound because, 2,3-butanediol can trigger the greatest level of growth promotion in plants. (Ryu et al., 2003). A number of antifungal compounds were also detected including 1-decanol and 2-hexen-1-ol which suppress the growth of the plant pathogenic fungi (Archbold et al., 2003 and Kai et al., 2009). A Gas chromatogram (Chromatogram is the visual representation of a chemical compound) of volatiles from the commercial rhizobacterial strain is shown in Figure 2.

Gas chromatogram of volatiles from the commercial rhizobacterial strain (Bacillus subtilis FZB24) where 2,3-butanediol (1) was found. (Identification of the chemical compound was confirmed by the comparison of the retention time (retention time: time taken for a chemical compound to come out of the gas chromatograph) of the chromatographic peak (1) to a sample pure chemical compound. 


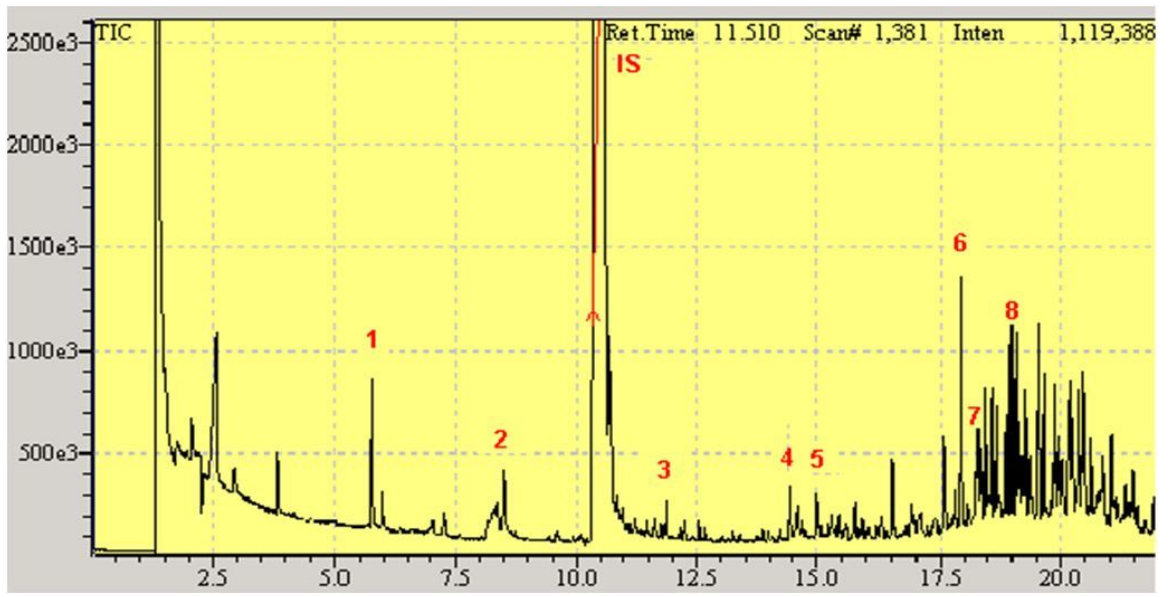

Figure 2: Gas chromatogram of volatiles from the commercial rhizobacterial strain

\section{Conclusion}

Rhizobacteria that promote plant growth and yield have gained worldwide importance in recent years and offer an attractive way to reduce the use of chemical fertilisers. The selection of novel and efficient PGPRs which promote plant growth and control plant diseases is very important in the promotion of sustainable agriculture, environmental and human safety and for the preservation of biodiversity. SPME-GC/MS profiling of rhizobacterial volatiles detected different volatile blends including the growth promoting VOC i.e., 2,3 butanediol. Further research will be carried out on different rhizobacterial strains (isolated from Andean potato rhizosphere) at the laboratory level for the early detection and selection of novel PGPRs through characterization of an array of their volatile organic compounds. The results from this project (VALORAM) will directly benefit to farmers by using natural microbial resources as inputs to improve production of high quality potato crops.

Siva Linga Sasanka.Velivelli is a PhD student in the School of Biological, Earth and Environmental Sciences, under the supervision of Dr Barbara Doyle Prestwich. The author wishes to acknowledge funding from European Community's Seventh Framework Programme FP7/20072013 (Grant No: 227522, 01/02/2009-31/01/2014). More information can be found on VALORAM website: http://valoram.ucc.ie 